\title{
An H I survey of highly flattened, edge-on, pure disk galaxies
}

L.D. Matthews ${ }^{1}$ and W. van Driel $^{2}$

${ }^{1}$ National Radio Astronomy Observatory, 520 Edgemont Road, Charlottesville, VA 22903, U.S.A. e-mail: lmatthew@nrao.edu

2 Unité Scientifique Nançay, CNRS B704, Observatoire de Paris, 5 place Jules Janssen, 92195 Meudon, France

Astron. Astrophys. Suppl. Ser. 143 (2000) 421-456

In the paper "An H I survey of highly flattened, edge-on, pure disk galaxies" by Matthews \& van Driel (A\&AS 143, 421), the spectra shown in Fig. 2 have their velocity axes plotted using the radio velocity convention $V=c\left(\nu_{0}-\nu\right) / \nu_{0}$ rather than the optical convention $V=c\left(\nu_{0}-\nu\right) / \nu$, as stated in the caption. All spectral measurements presented in the paper were performed using the optical velocity convention and are unaffected. However, the apparent velocity centroids of the plotted line profiles will show small offsets compared with the measured values quoted in the text. 